\title{
STRONG LAWS FOR BALANCED TRIANGULAR URNS
}

\author{
ARUP BOSE, ${ }^{* *}$ \\ AMITES DASGUPTA $* * * *$ AND \\ KRISHANU MAULIK, ${ }^{* * * * *}$ Indian Statistical Institute
}

\begin{abstract}
Consider an urn model whose replacement matrix is triangular, has all nonnegative entries, and the row sums are all equal to 1 . We obtain strong laws for the counts of balls corresponding to each color. The scalings for these laws depend on the diagonal elements of a rearranged replacement matrix. We use these strong laws to study further behavior of certain three-color urn models.
\end{abstract}

Keywords: Urn model; balanced triangular replacement matrix

2000 Mathematics Subject Classification: Primary 60G70; 60F05

Secondary $60 \mathrm{~F} 10$

\section{Introduction}

Consider an urn with balls of $(K+1)$ colors. Initially, the counts of balls of each color are nonrandom, strictly positive real numbers and the total count of balls in the urn equals 1 . Let the row vector $\boldsymbol{C}_{0}$ denote the initial count of balls of each color. The composition of the urn evolves by adding balls of different colors at times $n=1,2,3, \ldots$ as follows.

Suppose that $\boldsymbol{R}=\left(\left(r_{i j}\right)\right)$ is a $(K+1) \times(K+1)$ nonrandom balanced (that is, each row sum is the same and, hence, without loss of generality, equal to 1) replacement matrix with nonnegative entries. Let $\boldsymbol{C}_{n}$ denote the row vector of the counts of balls of each color after the $n$th trial, $n=1,2, \ldots$. At the $n$th trial, a ball is drawn at random from the urn with the current composition $\boldsymbol{C}_{n-1}$, so that the $i$ th color appears with probability $\boldsymbol{C}_{n-1, i} / n, i=1, \ldots,(K+1)$. If the $i$ th color appears then, for $j=1, \ldots,(K+1), r_{i j}$ balls of the $j$ th color are added to the urn before the next draw, together with the drawn ball. It is of interest to study the stochastic behavior of $\boldsymbol{C}_{n}$ as $n \rightarrow \infty$.

In the case when $\boldsymbol{R}$ is irreducible, let $\pi_{\boldsymbol{R}}$ be the unique stationary distribution satisfying $\boldsymbol{\pi}_{\boldsymbol{R}} \boldsymbol{R}=\boldsymbol{\pi}_{\boldsymbol{R}}$. Then (see, for example, Gouet (1997)) $\boldsymbol{C}_{n} /(n+1) \rightarrow \boldsymbol{\pi}_{\boldsymbol{R}}$ almost surely. Note that $\pi_{\boldsymbol{R}}$ is also a left eigenvector of $\boldsymbol{R}$ corresponding to the eigenvalue 1 . However, when $\boldsymbol{R}$ is not irreducible or balanced, the number of balls of different colors may increase at different rates and strong or weak limits for $\boldsymbol{C}_{n}$ are not known in full generality.

Janson (2006) considered two-color triangular urn models, where the replacement matrix was not necessarily balanced, and identified the weak limits of $\boldsymbol{C}_{n}$ in all possible cases. $\mathrm{He}$ mentioned urns with more colors and triangular replacement matrices as possible objects of further study (cf. Janson (2006, Problem 1.16)). Flajolet et al. (2006) considered a three-color

Received 15 August 2008; revision received 6 March 2009.

* Postal address: Stat-Math Unit, Indian Statistical Institute, 203 B. T. Road, Kolkata 700108, India.

** Email address: abose@isical.ac.in

*** Email address: amites@isical.ac.in

**** Email address: krishanu@ isical.ac.in 
urn having balanced triangular replacement matrix $\boldsymbol{R}$ with further conditions on the entries and obtained weak limit theorems.

Motivated by these results, we consider balanced, triangular urns with an arbitrary (but finite) number of colors. This assumption of balancedness on $\boldsymbol{R}$ allows us convenient application of martingale techniques. In contrast, Janson (2006) used the theory of branching processes and Flajolet et al. (2006) used generating functions. However, the application of martingale techniques to the study of urn models is not new; see, for example, Gouet (1997) and Bai and $\mathrm{Hu}$ (1999).

With appropriate scalings, we establish almost-sure convergence of each color count to a nonzero limit. Under an additional assumption, see (2.2), the limits are expressed in terms of the limits of certain martingales and left eigenvectors of appropriate submatrices of $\boldsymbol{R}$. These strong laws for urn models with an arbitrary but finite number of colors and balanced triangular replacement matrices are the main contributions of this paper.

The outline of the rest of the paper is as follows. In Section 2 we first describe a rearrangement of colors which converts any triangular balanced replacement matrix to an appropriate standard form. Our results are better described with reference to this standard form. Of course, the convergence holds without assuming the standard form, but then the rates are indirectly identified only through an algorithmic approach; see Remark 3.3. We also state the additional assumption (2.2) required to identify the limits in somewhat explicit forms. In this section we also establish the notation used to describe the limits and state some necessary auxiliary results.

In Section 3 we state and prove the main theorem. For a color whose corresponding diagonal entry is larger than all the preceding entries, we consider the right eigenvector of $\boldsymbol{R}$ corresponding to this eigenvalue and normalize the corresponding linear combination to obtain a martingale. This martingale turns out to be $L^{2}$-bounded and, hence, converges almost surely. The convergence of the individual color count then follows, since earlier colors have lower rates. For colors whose corresponding diagonal entry is not larger than the previous entries, we first show that the appropriately scaled color count is $L^{1}$-bounded. Then we form the appropriate martingale and obtain the convergence.

In Section 4 we analyze the three-color urn model with triangular replacement matrix as a corollary and obtain the asymptotic behavior of linear combinations of color counts. This gives an indication of further results that can be proved using the strong laws of this paper.

\section{Notation and preliminary results}

Suppose that $\boldsymbol{R}$ is a balanced triangular replacement matrix with row sums equal to 1 . Denote the diagonal elements of $\boldsymbol{R}$ as $r_{k}, 1 \leq k \leq K+1$. Let $1=i_{1}<i_{2}<\cdots<$ $i_{J}<i_{(J+1)}(=K+1)$ denote the indices of the running maxima of the diagonals, namely, $r_{1}=r_{i_{1}} \leq r_{i_{2}} \leq \cdots \leq r_{i_{J}} \leq r_{i_{(J+1)}}=r_{K+1}$ and, for $i_{j}<k<i_{(j+1)}$, we have $r_{k}<r_{i_{j}}$ for $j=1,2, \ldots, J$.

Remark 2.1. Since the row sums are equal to 1 and the elements of $\boldsymbol{R}$ are nonnegative, all the diagonal elements will be less than or equal to 1 . Thus, $(K+1)$ will always be an index of the running maximum of the diagonals.

The running maxima of $\boldsymbol{R}$ also leads to the following concepts.

Definition 2.1. Suppose that $\boldsymbol{R}$ is a balanced triangular replacement matrix. For $j=1,2, \ldots$, $J$, the colors indexed by $i_{j}, i_{j}+1, \ldots, i_{(j+1)}-1$ constitute the $j$ th block of colors, $i_{j}$ is called its leading index, and the corresponding color is called the leading color of the $j$ th block. The 
color indexed by $(K+1)$ will be the leading color and the sole constituent of the $(J+1)$ th block.

The triangular replacement matrix $\boldsymbol{R}$ with the indices of the running maxima of the diagonals can be visualized as

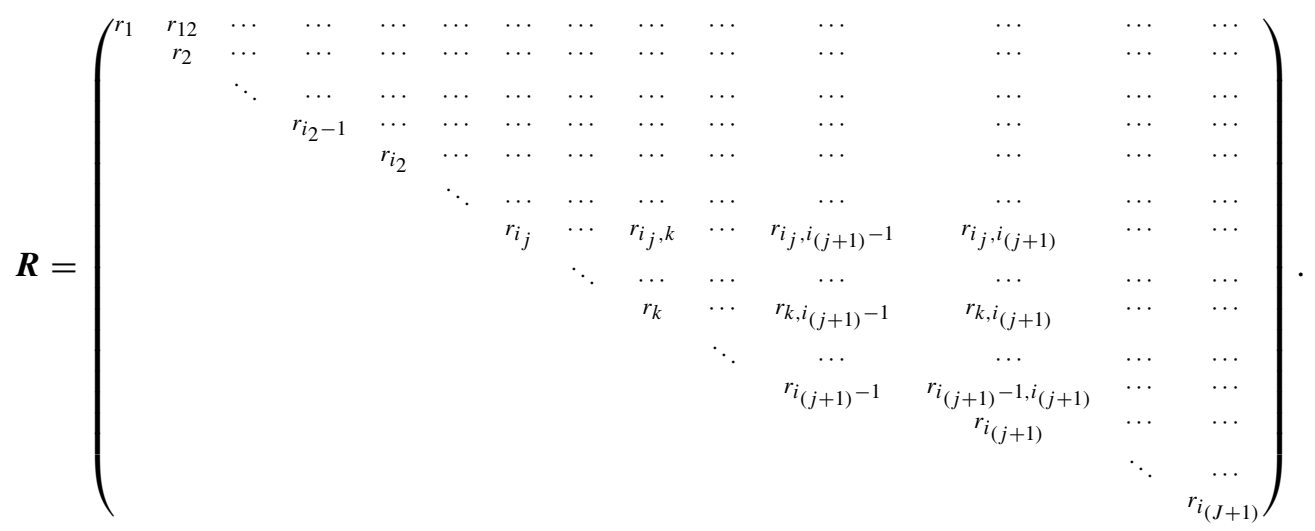

Here $r_{1}=r_{i_{1}} \leq r_{i_{2}} \leq \cdots \leq r_{i_{j}} \leq r_{i_{(j+1)}}=\leq \cdots \leq r_{i_{(J+1)}}$ give the running maxima of the diagonal entries. It will be helpful to study the concepts of rearrangement and blocks, while keeping this visualization in mind.

To study urn models with triangular replacement matrices, we need to arrange the colors systematically, which we describe next. This particular rearrangement keeps the replacement matrix triangular. The new replacement matrix is obtained by pre- and post-multiplication of $\boldsymbol{R}$ by permutation matrices. Thus, it remains balanced with row sum 1 and has the same set of eigenvalues. The elements of the new eigenvectors are also suitable rearrangements of the original elements.

Definition 2.2. The colors are said to be arranged in the increasing order if $\boldsymbol{R}$ satisfies the following: with $1=i_{1}<i_{2}<\cdots<i_{J}<i_{(J+1)}(=K+1)$ as the indices of the running maxima of the diagonals, for $i_{j}<k<i_{(j+1)}, j=1,2, \ldots, J$, we have

$$
\sum_{m=i_{j}}^{k-1} r_{m k}>0
$$

It is easy to see that (2.1) is equivalent to the fact that, for any nonleading color with index $k$ in the $j$ th block, namely, for $i_{j}<k<i_{(j+1)}, j=1,2, \ldots, J$, the part of the $k$ th column in the $j$ th block has at least one nonzero entry. Also, note that condition (2.1) holds only for nonleading colors.

The next proposition shows that any urn model with triangular replacement matrix can be transformed into another urn model with a triangular replacement matrix such that the colors are in increasing order.

Proposition 2.1. Suppose that $\boldsymbol{R}$ is a balanced triangular replacement matrix with row sums equal to 1 . Then there exists a rearrangement of colors into the increasing order, such that the replacement matrix remains triangular. 
Proof. From Remark 2.1, the $(K+1)$ th color forms the last block as required. We will now construct the other blocks inductively going backward. Within a block, the construction will move forward.

Suppose that we have constructed some blocks. If the leading index of the last constructed block, say $i$, is 1 , we are done.

If $i>1$, we construct the next (previous) block as follows. Let $k<i$ be such that $r_{k}=\max \left\{r_{m}: m<i\right\}$. The color with index $k$ is declared to be the leading color of the present block under construction and the index of this color may change, as discussed later, through rearrangement.

By our choice of leading colors, the diagonal entries of the indices of the leading colors will be in nondecreasing order, as required.

Next we decide which of the intermediate colors with index $m, k<m<i$, will be in the present block. This will be done through a process of rearrangement described inductively going forward.

Suppose that $l$ colors, including the leading color, satisfying (2.1), have already been obtained through rearrangement for the present block and the index of the leading color has changed to $k^{\prime}(>k)$ after this rearrangement. Then the index of the last considered color was $k^{\prime}+l-1$. If $k^{\prime}+l=i$, we have considered all intermediate colors and the construction of the block is over.

If $k^{\prime}+l<i$, consider the color with index $k^{\prime}+l$. By our choice of the leading color of the present block, we must have $r_{k^{\prime}}>r_{k^{\prime}+l}$. If we have $\sum_{m=k^{\prime}}^{k^{\prime}+l-1} r_{m,\left(k^{\prime}+l\right)}>0$, we take the color with index $k^{\prime}+l$ as the $(l+1)$ th color of the present block.

Otherwise, $r_{m,\left(k^{\prime}+l\right)}=0$ for $m=k^{\prime}, k^{\prime}+1, \ldots, k^{\prime}+l-1$. In this case we reshuffle the colors to bring the $\left(k^{\prime}+l\right)$ th color ahead of the $k^{\prime}$ th one, and then $r_{m,\left(k^{\prime}+l\right)}, m=k^{\prime}, k^{\prime}+1, \ldots, k^{\prime}+l-1$, will be the only entries that will move below the diagonal of the $k^{\prime}$ th column in the reshuffled replacement matrix. Hence, the reshuffled replacement matrix will remain triangular. After reshuffle, this color will have index $k^{\prime}$ and the index of the colors already in the present block will increase by 1 , with the present leading index increasing to $k^{\prime}+1$. The number of colors in the present block will remain at $l$. This gives the forward induction step for constructing a block. Since a color is shuffled if it fails to satisfy (2.1), all the remaining colors will satisfy this condition. Thus, we complete the backward induction step for the rearrangement of blocks.

In view of the above proposition, we will always assume, unless otherwise mentioned, that the colors are indeed in increasing order.

Note that if $r_{i_{j}}=r_{i_{j+1}}$ and $r_{m, i_{(j+1)}}=0$ for all $m=i_{j}, i_{j}+1, \ldots, i_{(j+1)}-1$, then we can reshuffle the colors to bring the $i_{(j+1)}$ th color ahead of the $i_{j}$ th one, yet maintaining the triangular structure of the replacement matrix and the increasing order of the colors. Hence, the rearrangement of colors into the increasing order will not be unique. To make the above rearrangement of colors into the increasing order a unique one, we further assume that

$$
\sum_{m=i_{j}}^{i_{(j+1)}-1} r_{m, i_{(j+1)}}>0 \quad \text { whenever } r_{i_{j}}=r_{i_{(j+1)}} .
$$

Assumption (2.2) is equivalent to requiring that there is at least one nonzero entry in the $i_{(j+1)}$ th column in the part corresponding to the $j$ th block. Its significance is discussed in Remark 3.1, below. 
We define the following submatrices and vectors corresponding to different blocks of colors.

Definition 2.3. Let $\boldsymbol{R}^{(j)}$ be the submatrix formed by the rows and columns corresponding to the indices of the $j$ th block. We will also write $\lambda_{j}=r_{i_{j}}=r_{1}^{(j)}$. The part of the vector $\boldsymbol{C}_{n}$ corresponding to the $j$ th block will be denoted by $\boldsymbol{C}_{n}^{(j)}$. Finally, $\boldsymbol{\rho}^{(j)}$ will denote the part of the $i_{j+1}$ th column corresponding to the $j$ th block.

By the definition of a block, $r_{i_{j}}=\lambda_{j}$ is the strictly largest eigenvalue of $\boldsymbol{R}^{(j)}$ and has multiplicity 1 . Let $\boldsymbol{\pi}^{(j)}$ be the unique left eigenvector of $\boldsymbol{R}^{(j)}$ corresponding to the eigenvalue $\lambda_{j}$ normalized so that its first element is 1 . Then $\pi^{(j)}$ satisfies

$$
\boldsymbol{\pi}^{(j)} \boldsymbol{R}^{(j)}=\lambda_{j} \boldsymbol{\pi}^{(j)}, \quad \boldsymbol{\pi}_{1}^{(j)}=1 .
$$

Observe that if $i_{j} \leq m, k<i_{(j+1)}$ then $C_{n m}=C_{n,\left(m-i_{j}+1\right)}^{(j)}$ and $r_{m k}=r_{\left(m-i_{j}+1\right)}^{(j)},\left(k-i_{j}+1\right)$. Here $\boldsymbol{\pi}_{k}^{(j)}, \boldsymbol{C}_{n k}$, and $\boldsymbol{C}_{n k}^{(j)}$ denote the $k$ th coordinate of $\boldsymbol{\pi}^{(j)}, \boldsymbol{C}_{n}$, and $\boldsymbol{C}_{n}^{(j)}$, respectively.

Next we define an index to count the number of times the diagonal entry corresponding to a leading color has occurred before. This is important in obtaining the rates of convergence for the color counts in Theorem 3.1 .

Definition 2.4. For the $j$ th block with leading color index $i_{j}$, let

$$
v_{j}=\#\left\{m: r_{m}=\lambda_{j}, m<i_{j}\right\} .
$$

Observe that, if $r_{m}=\lambda_{j}$ for some $m<i_{j}$ then $m$ is a leading index as well. So, if it is the first time a diagonal has value $\lambda_{j}$, we have $v_{j}=0$. Also, note that $\lambda_{j-1}=\lambda_{j}$ if and only if $v_{j}>0$, and in this case, $v_{j-1}=v_{j}-1$ holds.

The following useful result is obtained as a consequence of the above definitions.

Lemma 2.1. If the colors are in increasing order and the replacement matrix $\boldsymbol{R}$ is triangular, then all the coordinates of the vector $\pi^{(j)}$ are positive.

Proof. We prove this by induction on the coordinates of the vector.

Observe that if $r_{i_{j}}=0$ then the $j$ th block has only one color, namely, the $i_{j}$ th color. By the choice of normalization, $\boldsymbol{\pi}_{1}^{(j)}=1$. If $r_{i_{j}}=0$ then, by the above observation, the proof is complete. So, without loss of generality, we can take $r_{i_{j}}>0$.

Now assume that the first $k\left(<i_{(j+1)}-i_{j}\right)$ coordinates of $\boldsymbol{\pi}^{(j)}$ are positive. By the property of the eigenvector of $\boldsymbol{R}^{(j)}$, we have $\sum_{m=1}^{k+1} \boldsymbol{\pi}_{m}^{(j)} r_{\left(m+i_{j}-1\right),\left(k+i_{j}\right)}=r_{i_{j}} \boldsymbol{\pi}_{k+1}^{(j)}$. This gives

$$
\boldsymbol{\pi}_{k+1}^{(j)}=\frac{1}{r_{i_{j}}-r_{k+i_{j}}} \sum_{m=1}^{k} \boldsymbol{\pi}_{m}^{(j)} r_{\left(m+i_{j}-1\right),\left(k+i_{j}\right)} .
$$

The denominator on the right-hand side is positive, since $r_{i_{j}}$ is the strictly largest eigenvalue. By the induction hypothesis, $\boldsymbol{\pi}_{m}^{(j)}>0$ for $m=1, \ldots, k$. Also, by $(2.1), r_{\left(m+i_{j}-1\right),\left(k+i_{j}\right)}>0$ for some $m=1, \ldots, k$. This proves the induction step and the lemma.

We also denote $\Pi_{n}(s)=\prod_{i=0}^{n-1}(1+s /(i+1))$. Recall that Euler's formula for the gamma function gives

$$
\Pi_{n}(s) \sim \frac{n^{s}}{\Gamma(s+1)} \quad \text { if } s \text { is not a negative integer. }
$$




\section{Main results}

Now we state and prove our main result on the strong convergence of individual color counts.

Theorem 3.1. Suppose that $\boldsymbol{R}$ is a $(K+1) \times(K+1)$ balanced triangular matrix with row sums equal to 1 and $(J+1)$ blocks, and that the colors are in increasing order, satisfying (2.2). Then, for $j=1,2, \ldots, J+1$,

$$
\frac{C_{N}^{(j)}}{N^{\lambda_{j}}(\log N)^{v_{j}}} \rightarrow \boldsymbol{\pi}^{(j)} V_{j} \quad \text { almost surely and in } L^{2},
$$

where $V_{J+1}=1$. If $r_{1}=0$ then $V_{1}=C_{01}$. If $r_{1}>0$ then $V_{1}$ is a nondegenerate random variable. For $j=2,3, \ldots, J$, if $v_{j}=0$ then $V_{j}$ is also a nondegenerate random variable. If $v_{j}>0$, we further have

$$
V_{j}=\frac{1}{v_{j}} \boldsymbol{\pi}^{(j-1)} \boldsymbol{\rho}^{(j-1)} V_{j-1} .
$$

Remark 3.1. Owing to Lemma 2.1, the entries of $\boldsymbol{\pi}^{(j-1)}$ are all positive, and $\boldsymbol{\rho}^{(j-1)}$ has at least one positive entry by (2.2), which means that $\pi^{(j-1)} \boldsymbol{\rho}^{(j-1)}>0$ and, hence, recursively, all the $V_{j}$ s are nondegenerate.

Remark 3.2. If $r_{j}=1$ for some $j \leq K$ then the rest of the entries in the $j$ th row are 0 . Thus, (2.1) requires that $r_{j+1}=1$ and that the $(j+1)$ th color will be the leading color of a new block. However, if (2.2) is also assumed, even this is not possible. So, in the setup of Theorem 3.1, we must have $r_{j}<1$ for all $j \leq K$.

Remark 3.3. The rearrangement of colors into the increasing order and condition (2.2) help us identify the limits in Theorem 3.1. However, it will be clear from the proof that even without this assumption, appropriate strong laws hold. In this approach we do not use the concept of blocks. It can be shown that

$$
\frac{1}{N^{r_{1}}} \boldsymbol{C}_{N 1} \rightarrow W_{1} \quad \text { almost surely }
$$

for some random variable $W_{1}$. We can then inductively define the rates for all colors $j>1$ as follows: assume that, for all $1 \leq j \leq k$, there exist $s_{j}, \delta_{j}$, and random variables $W_{j}$ such that

$$
\frac{1}{N^{s_{j}}(\log N)^{\delta_{j}}} C_{N j} \rightarrow W_{j} \quad \text { almost surely. }
$$

If the part of the $(k+1)$ th column above the diagonal has all zero entries then, for some random variable $W_{k+1}$,

$$
\frac{1}{N^{r_{(k+1)}}} \boldsymbol{C}_{N,(k+1)} \rightarrow W_{k+1} .
$$

On the other hand, suppose that $r_{j,(k+1)}>0$ for some $j=1,2, \ldots, k$. Consider all the colors indexed by $j$ such that $r_{j,(k+1)}>0$. Let the highest rate of convergence for such color counts be $n^{s}(\log n)^{\delta}$. Then we can say that

$$
\frac{1}{a_{n}} \boldsymbol{C}_{N,(k+1)} \rightarrow W_{k+1} \quad \text { almost surely }
$$


for some random variable $W_{k+1}$, where

$$
a_{n}= \begin{cases}n^{s}(\log n)^{\delta} & \text { if } r_{k+1}<s \\ n^{s}(\log n)^{\delta+1} & \text { if } r_{k+1}=s \\ n^{r_{k+1}} & \text { if } r_{k+1}>s\end{cases}
$$

It is not clear whether we can easily write down how the $W_{k} \mathrm{~s}$ are related. However, under condition (2.2), if we rearrange the colors into the increasing order, the subvectors corresponding to each block obtained from the rearranged $W_{k}$ s are of course the same as the $\pi^{(j)} V_{j}$ s and will satisfy (3.1).

Proof of Theorem 3.1. The proof is through induction on the index of color $l$. Let $\chi_{n}$ be the row vector of order $(K+1)$ whose $m$ th entry is 1 if the $m$ th color is drawn at the $n$th draw and whose other entries are all 0 . Let $\mathcal{F}_{n}$ denote the $\sigma$-field generated by $\left\{\chi_{k}: 1 \leq k \leq n\right\}$.

We first quickly verify the result for $l=1$. If $r_{1}=0$ then the entire first column is 0 , so the first color count cannot change whichever color is drawn. Thus, $\boldsymbol{C}_{n 1}$ stays constant at $\boldsymbol{C}_{01}$ and the result is trivially true. Next consider $r_{1}>0$. In this case, we pool all the remaining colors, giving us the replacement matrix

$$
\left(\begin{array}{cc}
r_{1} & 1-r_{1} \\
0 & 1
\end{array}\right)
$$

Then the result for $l=1$ follows from Proposition 2.2(iii) of Bose et al. (2009).

Now assume that the result holds for the first $(l-1)$ colors for some $l \geq 2$. Suppose that the next color is the $k$ th color of the $j$ th block. Then we have $l=i_{j}+k-1$.

The following two observations follow from the induction hypothesis.

(i) If $l$ is a leading color, that is, $k=1$ and $l=i_{j}$, we have

$$
\frac{\boldsymbol{C}_{N m}}{N^{\lambda_{j-1}}(\log N)^{v_{j-1}}} \rightarrow \begin{cases}\boldsymbol{\pi}_{m+1-i_{(j-1)}}^{(j-1)} V_{j-1} & \text { if } i_{(j-1)} \leq m<i_{j}, \\ 0 & \text { if } m<i_{(j-1)},\end{cases}
$$

almost surely, as well as in $L^{2}$.

(ii) If $l$ is not a leading color, that is, $k>1$ and $l>i_{j}$, we have

$$
\frac{\boldsymbol{C}_{N m}}{N^{\lambda_{j}}(\log N)^{v_{j}}} \rightarrow \begin{cases}\boldsymbol{\pi}_{m+1-i_{j}}^{(j)} V_{j} & \text { if } i_{j} \leq m<l \\ 0 & \text { if } m<i_{j}\end{cases}
$$

almost surely, as well as in $L^{2}$. In particular, we have, for $m<l$,

$$
\mathrm{E}\left[\boldsymbol{C}_{N m}\right]= \begin{cases}O\left(N^{\lambda_{j-1}}(\log N)^{v_{j-1}}\right) & \text { if } k=1, \\ O\left(N^{\lambda_{j}}(\log N)^{v_{j}}\right) & \text { if } k>1 .\end{cases}
$$

We separate the proof into three cases: $k=1, v_{j}=0 ; k=1, v_{j}>0$; and $k>1$.

Case 1: $k=1$ and $v_{j}=0$. Let $\zeta$ be a right eigenvector of $\boldsymbol{R}$ for the eigenvalue $\lambda_{j}=r_{i_{j}}$, such that $\zeta_{i_{j}}=1$ and $\zeta_{k}=0$ for $k>i_{j}$. Observe that, since $\boldsymbol{R}$ is triangular, an eigenvector with the above conditions can be obtained by solving a triangular system of equations. Moreover, since $v_{j}=0$ gives $r_{k}<r_{i_{j}}$ for all $k<i_{j}$, we would further have $\zeta_{k} \geq 0$ for $k \leq i_{j}$. 
Consider the martingale $U_{N}=C_{N} \zeta / \Pi_{N}\left(\lambda_{j}\right)$. Then the martingale difference is

$$
U_{N+1}-U_{N}=\frac{\lambda_{j}}{\Pi_{N+1}\left(\lambda_{j}\right)}\left(\chi_{N+1}-\frac{C_{N}}{N+1}\right) \zeta .
$$

Denote by $\zeta^{2}$ the column vector whose coordinates are squares of those of $\zeta$. Hence, we have

$$
\begin{aligned}
\mathrm{E}\left[\left(U_{N+1}-U_{N}\right)^{2} \mid \mathcal{F}_{N}\right] & =\frac{\lambda_{j}^{2}}{\left(\Pi_{N+1}\left(\lambda_{j}\right)\right)^{2}}\left(\frac{\boldsymbol{C}_{N} \zeta^{2}}{N+1}-\left(\frac{\boldsymbol{C}_{N} \zeta}{N+1}\right)^{2}\right) \\
& \leq \frac{1}{\left(\Pi_{N+1}\left(\lambda_{j}\right)\right)^{2}} \frac{\boldsymbol{C}_{N} \zeta^{2}}{N+1} \\
& \leq \frac{\|\zeta\|_{\infty}}{(N+1) \Pi_{N+1}\left(\lambda_{j}\right)} U_{N} \\
& \leq\|\zeta\|_{\infty} \Gamma\left(\lambda_{j}+1\right) \frac{1+U_{N}^{2}}{(N+1)^{1+\lambda_{j}}}
\end{aligned}
$$

for all large enough $N$, where $\|\zeta\|_{\infty}$ is the largest coordinate of $\zeta$ (recall that all the coordinates of $\zeta$ are nonnegative), and the last inequality follows by using the fact that $2 U_{N} \leq 1+U_{N}^{2}$ and (2.3).

As in the proof of Proposition 2.2(iii) of Bose et al. (2009), this gives an iteration for $1+\mathrm{E}\left[U_{N}^{2}\right]$, and we can prove that $U_{N}$ is $L^{2}$-bounded and, hence, converges almost surely, as well as in $L^{2}$. Thus, by (2.3), $\boldsymbol{C}_{N} \zeta / N^{\lambda_{j}}$ also converges almost surely, as well as in $L^{2}$, to $V_{j}$, say.

Note that $U_{1}=\boldsymbol{C}_{1} \zeta /\left(1+\lambda_{j}\right)=\left(\boldsymbol{C}_{0} \zeta+\lambda_{j} \chi_{1} \zeta\right) /\left(1+\lambda_{j}\right)$. Since all the coordinates of $\boldsymbol{C}_{0}$ are positive, $\chi_{1}$ takes all coordinate vectors as values with positive probability. Thus, $\chi_{1} \zeta$ is constant if and only if all the coordinates of $\zeta$ are of the same value. This will be the case if and only if the corresponding eigenvalue is 1 , which, by Remark 3.2, holds if and only if $l=K+1$ and $j=J+1$. So in this case, $\boldsymbol{C}_{N} \boldsymbol{\zeta}=N+1$ and we have $U_{N}=\boldsymbol{C}_{N} \boldsymbol{\zeta} /(N+1)=1=V_{J+1}$.

If $j \leq J, U_{1}$ is nondegenerate and, hence, has positive variance. Since $U_{N}$ is a martingale, the variance of $U_{N}$ is nondecreasing and the limit variable has nonzero variance. So the limit variable $V_{j}$ is nondegenerate for $j \leq J$.

Finally, using the limit of $\boldsymbol{C}_{N} \zeta / N^{\lambda_{j}}$, since $\zeta_{k}=0$ for $k>i_{j}$ and $\zeta_{i_{j}}=1$, we have

$$
\lim _{N \rightarrow \infty} \frac{1}{N^{\lambda_{j}}} \boldsymbol{C}_{N 1}^{(j)}=V_{j}-\sum_{m=1}^{i_{j}-1} \zeta_{m} \lim _{N \rightarrow \infty} \frac{1}{N^{\lambda_{j}}} \boldsymbol{C}_{N m} \quad \text { almost surely and in } L^{2}
$$

provided that the limits on the right-hand side exist. Since $v_{j}=0$, we have $\lambda_{j-1}<\lambda_{j}$, and by (3.2), the limits on the right-hand side are all 0 . Thus,

$$
\frac{1}{N^{\lambda_{j}}} C_{N 1}^{(j)} \rightarrow V_{j} \quad \text { almost surely and in } L^{2},
$$

and $V_{j}$ is nondegenerate for $j \leq J, V_{J+1}=1$. Since $\pi_{1}^{(j)}=1$, we have proved the induction step for case 1.

For the other two cases, the proof is carried out in two steps. We first show the $L^{1}$-boundededness of $Z_{N}:=C_{N k}^{(j)} /\left(N^{\lambda_{j}}(\log N)^{v_{j}}\right)$ and then we show the required almost-sure convergence and the $L^{2}$-convergence by constructing an appropriate martingale. 
Step 1: $L^{1}$ bound. Observe that $\boldsymbol{C}_{N+1, l}=\boldsymbol{C}_{N l}+\sum_{m=1}^{l} \boldsymbol{\chi}_{N+1, m} r_{m l}$, which gives

$$
\mathrm{E}\left[\boldsymbol{C}_{N+1, l} \mid \mathcal{F}_{N}\right]=\boldsymbol{C}_{N l}\left(1+\frac{r_{l}}{N+1}\right)+\frac{1}{N+1} \sum_{m=1}^{l-1} \boldsymbol{C}_{N m} r_{m l},
$$

leading to

$$
\mathrm{E}\left[\boldsymbol{C}_{N+1, l}\right]=\mathrm{E}\left[\boldsymbol{C}_{N l}\right]\left(1+\frac{r_{l}}{N+1}\right)+\frac{1}{N+1} \sum_{m=1}^{l-1} \mathrm{E}\left[\boldsymbol{C}_{N m}\right] r_{m l} .
$$

Iterating, we have

$$
\mathrm{E}\left[\boldsymbol{C}_{N+1, l}\right]=\boldsymbol{C}_{0 l} \Pi_{N}\left(r_{l}\right)+\sum_{m=1}^{l-1} r_{m l} \sum_{n=0}^{N} \frac{1}{n+1} \mathrm{E}\left[\boldsymbol{C}_{n m}\right] \frac{\Pi_{N}\left(r_{l}\right)}{\Pi_{n}\left(r_{l}\right)} .
$$

To conclude, using $\boldsymbol{C}_{0 l}=\boldsymbol{C}_{0 k}^{(j)}$ and $\boldsymbol{C}_{N l}=\boldsymbol{C}_{N k}^{(j)}$,

$$
\frac{\mathrm{E}\left[\boldsymbol{C}_{N k}^{(j)}\right]}{\Pi_{N}\left(r_{l}\right)}=\boldsymbol{C}_{0 k}^{(j)}+\sum_{m=1}^{l-1} r_{m l} \sum_{n=0}^{N-1} \frac{1}{n+1} \frac{\mathrm{E}\left[\boldsymbol{C}_{n m}\right]}{\Pi_{n}\left(r_{l}\right)} .
$$

Case 2: $k=1$ and $v_{j}>0$. We also have $v_{j-1}=v_{j}-1$ and $r_{l}=\lambda_{j}=\lambda_{j-1}$. Then using (2.3), (3.4), and (3.6), we have

$$
\begin{aligned}
\frac{\mathrm{E}\left[\boldsymbol{C}_{N k}^{(j)}\right]}{\Pi_{N}\left(\lambda_{j}\right)} & =\boldsymbol{C}_{0 k}^{(j)}+\sum_{m=1}^{l-1} r_{m l} \sum_{n=0}^{N-1} \frac{(\log (n+2))^{v_{j-1}}}{n+1} \frac{\mathrm{E}\left[\boldsymbol{C}_{n m}\right]}{n^{\lambda_{j}}(\log (n+2))^{v_{j}-1}} \frac{n^{r_{l}}}{\Pi_{n}\left(r_{l}\right)} \\
& =O\left((\log N)^{v_{j}}\right) .
\end{aligned}
$$

Thus, again using (2.3), $\left\{Z_{N}\right\}$ becomes $L^{1}$-bounded.

Case 3: $k>1$. Here we have $r_{l}<\lambda_{j}$. Then using (2.3), (3.4), and (3.6), we have

$$
\begin{aligned}
\frac{\mathrm{E}\left[\boldsymbol{C}_{N k}^{(j)}\right]}{\Pi_{N}\left(r_{l}\right)} & =\boldsymbol{C}_{0 k}^{(j)}+\sum_{m=1}^{l-1} r_{m l} \sum_{n=0}^{N-1} \frac{(\log (n+2))^{v_{j}}}{(n+1) n^{r_{l}-\lambda_{j}}} \frac{\mathrm{E}\left[\boldsymbol{C}_{n m}\right]}{n^{\lambda_{j}}(\log (n+2))^{v_{j}}} \frac{n^{r_{l}}}{\Pi_{n}\left(r_{l}\right)} \\
& =O\left(N^{\lambda_{j}-r_{l}}(\log N)^{v_{j}}\right) .
\end{aligned}
$$

Thus, again using (2.3), $\left\{Z_{N}\right\}$ becomes $L^{1}$-bounded.

Step 2: convergence. Now we construct the relevant martingale. Using (3.5), it is easy to check that

$$
M_{N}=\frac{C_{N l}}{\Pi_{N}\left(r_{l}\right)}-\sum_{m=1}^{l-1} \sum_{n=0}^{N-1} \frac{r_{m l}}{n+1+r_{l}} \frac{C_{n m}}{\Pi_{n}\left(r_{l}\right)}
$$

forms a martingale. The corresponding martingale difference is given by

$$
M_{N+1}-M_{N}=\frac{1}{\Pi_{N+1}\left(r_{l}\right)} \sum_{m=1}^{l}\left(\chi_{(N+1), m}-\frac{C_{N m}}{N+1}\right) r_{m l},
$$


which leads to, using (3.4) and the $L^{1}$-boundedness of $Z_{N}$,

$$
\begin{aligned}
\mathrm{E}\left[\left(M_{N+1}-M_{N}\right)^{2}\right] & =\frac{1}{\left(\Pi_{N+1}\left(r_{l}\right)\right)^{2}} \mathrm{E}\left[\frac{\sum_{m=1}^{l} \boldsymbol{C}_{N m} r_{m l}^{2}}{N+1}-\left(\frac{\sum_{m=1}^{l} \boldsymbol{C}_{N m} r_{m l}}{N+1}\right)^{2}\right] \\
& \leq \frac{1}{\left(\Pi_{N+1}\left(r_{l}\right)\right)^{2}} \mathrm{E}\left[\frac{\sum_{m=1}^{l} \boldsymbol{C}_{N m} r_{m l}^{2}}{N+1}\right] \\
& =O\left(\frac{(\log N)^{v_{j}}}{N^{1+2 r_{l}-\lambda_{j}}}\right) .
\end{aligned}
$$

When $k=1, v_{j}>0$, and $\lambda_{j}=r_{l}=0$, we can further improve on the order of the squared moment of the martingale difference given in (3.9). Observe that $l$ being a leading color and $r_{l}=0$ imply that $r_{m}=0$ for all $m<l$, which makes each of the colors indexed by $m \leq l$ a leading color of a block of size 1 . This implies that $j=l$. Since the diagonal elements corresponding to all these colors are 0 , we have $v_{m}=m-1$ for $m \leq l$. Since $v_{j}=j-1=l-1>0$, we have $l \geq 2$. Thus, (3.4) simplifies to $\mathrm{E}\left[\boldsymbol{C}_{n m}\right]=O\left((\log n)^{m-1}\right)$ for $m<l$. Also, $r_{l}$ being 0 , the $l$ th term in the sum of (3.8) does not contribute. Hence, we have

$$
\mathrm{E}\left[\left(M_{N+1}-M_{N}\right)^{2}\right]=O\left(\frac{(\log N)^{\left(v_{j}-1\right)}}{N}\right) .
$$

Case 2: $k=1$ and $v_{j}>0$. Here we have $l=i_{j}$ and $r_{l}=\lambda_{j}$. First assume that $r_{l}=\lambda_{j}>0$. Then the right-hand side of (3.9) is summable. Hence, $M_{N}$ is an $L^{2}$-bounded martingale, which converges almost surely, as well as in $L^{2}$. Since $v_{j}>0$, we have

$$
\frac{1}{(\log N)^{v_{j}}} M_{N} \rightarrow 0 \text { almost surely and in } L^{2} .
$$

Next assume that $r_{l}=\lambda_{j}=0$. Then, using (3.10), we find that $M_{N} /(\log N)^{v_{j} / 2}$ is $L^{2}$-bounded. Hence, we have $M_{N} /(\log N)^{v_{j}} \rightarrow 0$ in $L^{2}$. We will now show that

$$
Y_{N}:=\frac{1}{(\log N)^{v_{j}}} M_{N} \quad \text { converges almost surely. }
$$

Since the $L^{2}$-limit is known to be 0 , we will then have $Y_{N} \rightarrow 0$ almost surely, as well as in $L^{2}$. With $\Delta_{N}=1 /(\log N)^{v_{j}}$, we have $Y_{N}=M_{N} \Delta_{N}$, which gives

$$
Y_{N+1}-Y_{N}=M_{N+1}\left(\Delta_{N+1}-\Delta_{N}\right)+\Delta_{N}\left(M_{N+1}-M_{N}\right) .
$$

Thus, it is enough to show that the partial sums of each of the terms on the right-hand side of (3.11) converges almost surely.

Now, $\Delta_{N}$ is a deterministic sequence and

$$
\left|\Delta_{N+1}-\Delta_{N}\right|=\Delta_{N+1}\left[\left(\frac{\log (N+1)}{\log N}\right)^{v_{j}}-1\right] \sim \frac{v_{j}}{N(\log N)^{v_{j}+1}} .
$$

We further know that $M_{N} /(\log N)^{v_{j} / 2}$ is $L^{2}$-bounded and, hence, $L^{1}$-bounded. Thus, recalling that $v_{j}>0, \mathrm{E}\left[\left|M_{N+1}\left(\Delta_{N+1}-\Delta_{N}\right)\right|\right]$ is summable and, hence, the first term on the right-hand side of (3.11) is almost surely absolutely summable. 
Since $M_{N+1}-M_{N}$ is a martingale difference, so is the second term on the right-hand side of (3.11). By (3.10) we have

$$
\mathrm{E}\left[\Delta_{N}^{2}\left(M_{N+1}-M_{N}\right)^{2}\right]=O\left(\frac{1}{(\log N)^{2 v_{j}}} \frac{(\log N)^{v_{j}-1}}{N}\right),
$$

which is summable, as $v_{j}>0$. Hence, the second term on the right-hand side of (3.11) is the difference sequence of a martingale which converges almost surely, as well as in $L^{2}$. Thus, we find that $Y_{N}$ converges almost surely, as well as in $L^{2}$, to 0 even when $r_{l}=\lambda_{j}=0$. Hence, under the assumption of case $2, k=1$ and $v_{j}>0$,

$$
\frac{1}{(\log N)^{v_{j}}} M_{N} \rightarrow 0 \quad \text { almost surely and in } L^{2} .
$$

Using (3.7), we then have

$$
\begin{aligned}
& \lim _{N \rightarrow \infty} \frac{\boldsymbol{C}_{N, i_{j}}}{N^{\lambda_{j}}(\log N)^{v_{j}}} \\
& \quad=\lim _{N \rightarrow \infty} \frac{\Pi_{N}\left(\lambda_{j}\right)}{N^{\lambda_{j}}(\log N)^{v_{j}}} \sum_{m=1}^{i_{j}-1} \sum_{n=0}^{N-1} r_{m, i_{j}} \frac{(\log n)^{v_{j}-1}}{n+1+\lambda_{j}} \frac{n^{\lambda_{j}}}{\Pi_{n}\left(\lambda_{j}\right)} \frac{\boldsymbol{C}_{n m}}{n^{\lambda_{j}}(\log n)^{v_{j}-1}},
\end{aligned}
$$

where the limit is in the almost-sure as well as the $L^{2}$ sense. Since $v_{j}>0$, we have $\lambda_{j}=\lambda_{j-1}$ and $v_{j}-1=v_{j-1}$. Thus, from (2.3) and (3.2), we have

$$
\begin{aligned}
\frac{C_{N 1}^{(j)}}{N^{\lambda_{j}}(\log N)^{v_{j}}} & =\frac{\boldsymbol{C}_{N i_{j}}}{N^{\lambda_{j}}(\log N)^{v_{j}}} \\
& \rightarrow \frac{1}{v_{j}} \sum_{m=1}^{i_{j}-i_{(j-1)}} \boldsymbol{\pi}_{m}^{(j-1)} \boldsymbol{\rho}_{m}^{(j-1)} V_{j-1} \\
& =\frac{1}{v_{j}} \pi^{(j-1)} \boldsymbol{\rho}^{(j-1)} V_{j-1} \quad \text { almost surely and in } L^{2} .
\end{aligned}
$$

We obtain the formula for $V_{j}$ in terms of $V_{j-1}$ from (3.12). Since, by normalization, $\boldsymbol{\pi}_{1}^{(j)}=1$, we have proved the induction step for case 2 .

Case 3: $k>1$. Here $r_{l}<\lambda_{j}$ holds and, hence, $\lambda_{j}>0$. If $r_{l}>\lambda_{j} / 2$, using (3.9), $M_{N}$ is an $L^{2}$-bounded martingale and, hence, converges almost surely, as well as in $L^{2}$. Thus,

$$
\frac{M_{N}}{N^{\lambda_{j}-r_{l}}(\log N)^{v_{j}}} \rightarrow 0 \quad \text { almost surely and in } L^{2} .
$$

The analysis is a bit more elaborate when $r_{l} \leq \lambda_{j} / 2$. If $r_{l}=\lambda_{j} / 2$ then, using (3.9), $M_{N} /(\log N)^{\left(v_{j}+1\right) / 2}$ is $L^{2}$-bounded. On the other hand, if $r_{l}<\lambda_{j} / 2$, again using (3.9), $M_{N} /\left(N^{\lambda_{j} / 2-r_{l}}(\log N)^{v_{j} / 2}\right)$ is $L^{2}$-bounded. Hence, for $r_{l} \leq \lambda_{j} / 2$,

$$
\frac{M_{N}}{N^{\lambda_{j}-r_{l}}(\log N)^{v_{j}}} \rightarrow 0 \quad \text { in } L^{2}
$$

We will now show that $Y_{N}:=M_{N} /\left\{N^{\lambda_{j}-r_{l}}(\log N)^{v_{j}}\right\}$ converges almost surely (to 0$)$ even when $r_{l} \leq \lambda_{j} / 2$. With $\Delta_{N}=1 /\left\{N^{\lambda_{j}-r_{l}}(\log N)^{v_{j}}\right\}$, we have $Y_{N}=M_{N} \Delta_{N}$, which gives

$$
Y_{N+1}-Y_{N}=M_{N+1}\left(\Delta_{N+1}-\Delta_{N}\right)+\Delta_{N}\left(M_{N+1}-M_{N}\right) .
$$


As before, it is enough to show that the partial sums of each of the terms on the right-hand side converge almost surely, which can be proved in a similar manner using

$$
\left|\Delta_{N+1}-\Delta_{N}\right|=\Delta_{N+1}\left[\left(1+\frac{1}{N}\right)^{\lambda_{j}-r_{l}}\left(\frac{\log (N+1)}{\log N}\right)^{v_{j}}-1\right] \sim \frac{\lambda_{j}-r_{l}}{N^{1+\lambda_{j}-r_{l}}(\log N)^{v_{j}}} .
$$

We leave the details to the reader.

Using (3.7), we have

$$
\begin{aligned}
& \lim _{N \rightarrow \infty} \frac{\boldsymbol{C}_{N l}}{N^{\lambda_{j}}(\log N)^{v_{j}}} \\
& \quad=\lim _{N \rightarrow \infty} \frac{\Pi_{N}\left(r_{l}\right)}{N^{\lambda_{j}}(\log N)^{v_{j}}} \sum_{m=1}^{l-1} \sum_{n=0}^{N-1} r_{m l} \frac{(\log n)^{v_{j}}}{\left(n+1+r_{l}\right) n^{r_{l}-\lambda_{j}}} \frac{n^{r_{l}}}{\Pi_{n}\left(r_{l}\right)} \frac{\boldsymbol{C}_{n m}}{n^{\lambda_{j}}(\log n)^{v_{j}}},
\end{aligned}
$$

where the limit is in the almost-sure as well as the $L^{2}$ sense. Thus, from (3.3), using the fact that $\boldsymbol{\pi}^{(j)}$ is the left eigenvector of $\boldsymbol{R}^{(j)}$ for the eigenvalue $\lambda_{j}$, we have

$$
\begin{aligned}
\frac{\boldsymbol{C}_{N k}^{(j)}}{N^{\lambda_{j}}(\log N)^{v_{j}}} & =\frac{\boldsymbol{C}_{N l}}{N^{\lambda_{j}}(\log N)^{v_{j}}} \\
& \rightarrow \frac{1}{\lambda_{j}-r_{l}} \sum_{m=1}^{k-1} \boldsymbol{\pi}_{m}^{(j)} r_{m k}^{(j)} V_{j} \\
& =\boldsymbol{\pi}_{k}^{(j)} V_{j} \quad \text { almost surely and in } L^{2} .
\end{aligned}
$$

This completes the proof of the induction step and the proof of the theorem.

\section{Three-color urns}

We now specialize to three-color urns. The replacement matrix is then

$$
\boldsymbol{R}=\left(\begin{array}{ccc}
r_{11} & r_{12} & r_{13} \\
0 & r_{22} & r_{23} \\
0 & 0 & 1
\end{array}\right)
$$

We assume that the entries are nonnegative, that each row sum is equal to 1 , and that (2.2) holds. The latter is equivalent to assuming that $r_{11}<1, r_{22}<1$, and $r_{12}>0$, whenever $r_{11}=r_{22}$.

This three-color urn model has already been considered in Flajolet et al. (2006), who further assumed that $r_{11}>0, r_{12}>0$, and $r_{22}>0$. Under these assumptions, they established the weak convergence of appropriately scaled $\boldsymbol{C}_{n}$ and obtained the limit distributions (cf. Propositions 25 and 26 of Flajolet et al. (2006)).

In contrast, we have established the almost-sure convergence of scaled $\boldsymbol{C}_{n}$. We restate our result in a form that is applicable to the three-color urn.

Corollary 4.1. Suppose that we have a three-color urn model with triangular replacement matrix $\boldsymbol{R}$ given by (4.1) with nonnegative entries and each row sum equal to 1 . Assume that $r_{11}<1, r_{22}<1$, and $r_{12}>0$, whenever $r_{11}=r_{22}$. Then there exist nondegenerate random variables $V_{1}, V_{2}$, and $V_{3}$ such that the following assertions hold.

(i) $C_{n 3} / n \rightarrow 1$. 
(ii) If $r_{11}=0$ then $\boldsymbol{C}_{n 1}$ stays unchanged at $\boldsymbol{C}_{01}$. If $r_{11}>0$ then $\boldsymbol{C}_{n 1} / n^{r_{11}} \rightarrow V_{1}$.

(iii) If $r_{22}>r_{11}$ then $C_{n 2} / n^{r_{22}} \rightarrow V_{2}$.

(iv) If $r_{22}=r_{11}$ and $r_{12}>0$, then $C_{n 2} /\left(n^{r_{22}} \log n\right) \rightarrow r_{12} V_{1}$.

(v) If $r_{22}<r_{11}$ and $r_{12}>0$, then $C_{n 2} / n^{r_{11}} \rightarrow r_{12} V_{1} /\left(r_{11}-r_{22}\right)$. If $0<r_{22}<r_{11}$ and $r_{12}=0$, then $C_{n 2} / n^{r_{22}} \rightarrow V_{3}$.

(vi) If $r_{12}=r_{22}=0$ then $\boldsymbol{C}_{n 2}$ stays unchanged at $\boldsymbol{C}_{02}$.

The convergence of all the above random variables is almost sure as well as in $L^{2}$.

Three-color urn models with reducible and block triangular balanced replacement matrices were considered in Bose et al. (2009). They established the almost-sure convergence of appropriately scaled individual color counts as well as weak/strong limits of linear combinations $\boldsymbol{C}_{n} \boldsymbol{\zeta}$ for suitable vectors $\boldsymbol{\zeta}$ obtained from the Jordan decomposition of $\boldsymbol{R}$.

Armed with the strong laws obtained from Corollary 4.1, we can now extend the results of Bose et al. (2009) to the case of three-color urn models with triangular replacement matrices. Observe that $\xi_{1}=(1,0,0)^{\top}$ and $\xi_{3}=(1,1,1)^{\top}$ are always right eigenvectors of $\boldsymbol{R}$ with respect to the eigenvalues $r_{11}$ and $r_{33}$, respectively. Clearly, $\boldsymbol{C}_{n} \boldsymbol{\xi}_{3} /(n+1)=1$ for all $n$. Also, since $\boldsymbol{C}_{n} \boldsymbol{\xi}_{1}=\boldsymbol{C}_{n 1}$, its limiting behavior is given in Corollary 4.1(ii).

Now observe that if $r_{11} \neq r_{22}$ then $\boldsymbol{R}$ has a right eigenvector $\xi_{2}$ with respect to the eigenvalue $r_{22}$, given by $\xi_{2}=\left(r_{12}, r_{22}-r_{11}, 0\right)^{\top}$. If $r_{22}>r_{11}$ then, from Corollary 4.1(ii) and (iii), we have

$$
\frac{\boldsymbol{C}_{n} \boldsymbol{\xi}_{2}}{n^{r_{22}}} \rightarrow\left(r_{22}-r_{11}\right) V_{2} \text { almost surely and in } L^{2},
$$

since the contribution of $\boldsymbol{C}_{n 1}$ is of smaller order.

If $r_{22}<r_{11}$ and $r_{12}=0$, then observe that $\boldsymbol{C}_{n} \boldsymbol{\xi}_{2}=\left(r_{22}-r_{11}\right) \boldsymbol{C}_{n 2}$. If we further have $r_{22}>0$ then from Corollary 4.1(v) we obtain

$$
\frac{C_{n} \xi_{2}}{n^{r_{22}}} \rightarrow\left(r_{22}-r_{11}\right) V_{3} \quad \text { almost surely and in } L^{2} .
$$

But, if we have $r_{12}=r_{22}=0$ then $\boldsymbol{C}_{n} \boldsymbol{\xi}_{2}$ remains constant at $\boldsymbol{C}_{0} \boldsymbol{\xi}_{2}$.

If $0=r_{22}<r_{11}$ and $r_{12}>0$, then observe that, $\boldsymbol{\xi}_{2}$ being an eigenvector of $\boldsymbol{R}$ with respect to the eigenvalue $r_{22}=0, \boldsymbol{R} \xi_{2}$ becomes a null vector. Also, if, for $j=1,2,3$, the $j$ th color appears in the $n$th draw, $\boldsymbol{C}_{n} \boldsymbol{\xi}_{2}$ increases by an amount which is the $j$ th coordinate of $\boldsymbol{R} \boldsymbol{\xi}_{2}$, namely, 0 . Thus, $\boldsymbol{C}_{n} \boldsymbol{\xi}_{2}$ remains constant at $\boldsymbol{C}_{0} \boldsymbol{\xi}_{2}$.

The situation becomes interesting when $0<r_{22}<r_{11}$ and $r_{12}>0$. Note that in this case we find, from Corollary 4.1(ii) and (v), that $\boldsymbol{C}_{n} \xi_{2} / n^{r_{11}} \rightarrow 0$ almost surely, as well as in $L^{2}$. We summarize the asymptotic behavior of $\boldsymbol{C}_{n} \xi_{2}$ in this case in the following proposition.

Proposition 4.1. Suppose that we have a three-color urn model with triangular replacement matrix $\boldsymbol{R}$ given by (4.1) with nonnegative entries and each row sum equal to 1 . Assume that $0<r_{22}<r_{11}$ and $r_{12}>0$. Let $V_{1}$ be the almost-sure limit of $\boldsymbol{C}_{n 1} / n^{r_{11}}$ obtained in Corollary 4.1(ii). Then the following assertions hold.

(i) If $r_{22}<r_{11} / 2$ then

$$
\frac{\boldsymbol{C}_{n} \boldsymbol{\xi}_{2}}{\sqrt{n^{r_{11}}}} \stackrel{\mathrm{w}}{\rightarrow} N\left(0, \frac{r_{12} r_{22}^{2}\left(r_{12}+r_{11}-r_{22}\right)}{r_{11}-2 r_{22}} V_{1}\right) .
$$


(ii) If $r_{22}=r_{11} / 2$ then

$$
\frac{\boldsymbol{C}_{n} \xi_{2}}{\sqrt{n^{r_{11} \log n}}} \stackrel{\mathrm{w}}{\rightarrow} N\left(0, r_{12} r_{22}^{2}\left(r_{12}+r_{11}-r_{22}\right) V_{1}\right) .
$$

(iii) If $r_{22}>r_{11} / 2$ then $C_{n} \xi_{2} / n^{r_{22}}$ converges almost surely and in $L^{2}$ to a nondegenerate random variable.

Note that here $V_{1}$ is a random variable and the above limits are to be interpreted as variance mixtures of normal distributions.

Proof of Proposition 4.1. Observe that only the first two components of the eigenvector $\xi_{2}$ corresponding to the eigenvalue $r_{22}$ are nonzero in this case. Hence, the evolution equation

$$
\boldsymbol{C}_{n+1} \xi_{2}=\boldsymbol{C}_{n} \xi_{2}+r_{22} \chi_{n+1} \xi_{2}
$$

can be written in terms of the first two colors only as

$$
S_{n+1} \xi=S_{n} \xi+\lambda s \chi_{n+1} \xi,
$$

where $s=r_{11}, \lambda=r_{22} / r_{11}$, and $\boldsymbol{S}_{n}, \boldsymbol{\xi}$, and $\chi_{n}$ are the restrictions of $\boldsymbol{C}_{n}, \boldsymbol{\xi}_{2}$, and $\chi_{n}$ to the first two colors only, with some abuse of notation. From Corollary 4.1(ii) and (v), we have $S_{n} / n^{s} \rightarrow \pi V_{1}$ with probability 1 , where $\pi=\left(1, r_{12} /\left(r_{11}-r_{22}\right)\right)$. Now the analysis of the proof of Theorem 3.1(v)-(vii) of Bose et al. (2009) can be repeated verbatim, with obvious changes for the almost-sure limits of $S_{n} / n^{s}$ for the calculation of the limiting variances, which is outlined below.

The limiting variance in (i) above will be $r_{22}^{2} V_{1} \pi \xi^{2} /\left(r_{11}-2 r_{22}\right)$, where $\xi^{2}$ is a column vector with coordinates which are the squares of those of $\xi$. The limiting variance in (ii) above will be $r_{22}^{2} V_{1} \pi \xi^{2}$. A simplification in either case gives the result.

Finally, if $r_{12}>0$ and $r_{11}=r_{22}$, then, for this repeated eigenvalue, it can be checked that, for any $\alpha, \xi_{2}=\left(\alpha, 1 / r_{12}, 0\right)^{\top}$ is a Jordan vector satisfying $\boldsymbol{R} \xi_{2}=\xi_{1}+r_{11} \xi_{2}$. Hence, from Corollary 4.1(ii) and (iv), we have

$$
\frac{C_{n} \xi_{2}}{n^{r_{22}} \log n} \rightarrow V_{1} \quad \text { almost surely and in } L^{2},
$$

where $V_{1}$ is the almost-sure limit of $\boldsymbol{C}_{n 1} / n^{r_{11}}$ obtained in Corollary 4.1(ii).

\section{Acknowledgement}

We thank the anonymous referee for an extremely careful reading of the manuscript and highly constructive comments.

\section{References}

BAi Z. D. AND Hu, F. (1999). Asymptotic theorems for urn models with nonhomogeneous generating matrices. Stoch. Process. Appl. 80, 87-101.

Bose, A., Dasgupta, A. And Maulik, K. (2009). Multicolor urn models with reducible replacement matrices. Bernoulli 15, 279-295.

Flajolet, P., Dumas, P. and Puyhaubert, V. (2006). Some exactly solvable models of urn process theory. Discrete Math. Theoret. Computer Sci. AG, 59-118.

Gouet, R. (1997). Strong convergence of proportions in a multicolor Pólya urn. J. Appl. Prob. 34, 426-435.

Janson, S. (2006). Limit theorems for triangular urn schemes. Prob. Theory Relat. Fields 134, 417-452. 